Running Head: Attachment and ER

\title{
Associations between Attachment and Emotion-Specific Emotion Regulation with and without Relationship Insecurity Priming
}

\author{
Sarah J. Clear \\ Melanie J. Zimmer-Gembeck \\ School of Applied Psychology and Menzies Health Institute of Queensland \\ Griffith University
}




\begin{abstract}
Attachment theory and previous research on emotion regulation (ER) suggest that ER will be associated with adult attachment orientation, with the expectation of different associations of attachment avoidance, anxiety, and security with specific ER patterns. In addition, research has shown that the emotion under consideration and the context may matter to patterns of ER and associations between attachment and ER. In the present study, we examined associations between attachment representations, and emotion specific (sadness, worry, and anger) ER among late adolescents and young adults aged 16 to 23 years $(M=19.6, S D=1.58)$. In addition, to consider context, participants were randomly assigned to report ER following insecurity priming or no priming. Participants were 383 (181 young men, 202 young women) students who completed a self-report questionnaire. As expected, multivariate regression results examining all attachment orientations simultaneously showed that attachment anxiety was associated with greater dysregulation (sadness, worry and anger), but also more anger suppression. In contrast, attachment avoidance was associated with greater suppression (sadness and worry), but also more anger dysregulation. Attachment security was associated with less dysregulation (sadness, worry and anger), and less sadness and worry suppression. Finally, sadness and anger dysregulation were higher when reported after insecurity priming compared to the standard no prime condition, but few associations between attachment orientations and ER were moderated by condition. The results suggest that individuals' attachment representations are associated with ER, with security a benefit to adaptive ER, and anxiety and avoidance playing different roles in maladaptive ER for different emotions.
\end{abstract}

Keywords: Attachment, emotion regulation, specific emotions, interpersonal stress, priming effects. 


\section{Associations between Attachment and Emotion-Specific Emotion Regulation with and without Relationship Insecurity Priming}

Classic attachment theory (Bowlby, 1969), and contemporary extensions to this theory (Ainsworth, 1989; Cassidy, 1994; Main, 1996, 2000; Mikulincer \& Shaver, 2007; Shaver \& Mikulincer, 2002; Sroufe, 1996) have become important frameworks for understanding how a history of interactions with caregivers and important others (e.g., romantic partners) can provide safe and secure relationships that promote positive social and emotional adaptation across the lifespan. For example, a basic premise of classic developmental attachment theory (Bowlby, 1969; Sroufe, 1996) is that early interactions and experiences with primary caregivers form a critical context for concurrent and later emotion regulation (ER) and interpersonal interactions. Such views have been extended to attachment relationships formed in later life (Ainsworth, 1989; Mikulincer \& Shaver, 2007). Thus, one key tenet of attachment theories, whether the focus is on children, adolescents or adults, is that the quality of attachment orientation, sometimes referred to as attachment style, can explain individual differences in the management and regulation of emotions and responses in interpersonal situations (Mikulincer \& Shaver, 2007; Wei, Vogul, Ku, \& Zakalik, 2005).

Despite the theoretical importance of attachment for adaptive ER (Cassidy, 1994; Kopp, 1989; Mikulincer \& Shaver, 2007; Sroufe, 1996; Thompson, 1994) and despite multiple studies showing that adaptive ER or ER deficits can be meaningfully linked to attachment style (Cassidy, Shaver, Mikulincer, \& Lavy, 2009; Cooper, Shaver, \& Collins, 1998; Lopez \& Gormley, 2002), very little previous research has considered whether the particular emotion and the context may matter to specific ER responses (see Roque, Veríssimo, Fernandes, \& Rebelo, 2013 for an exception of a study with 
toddlers). In other words, it has been rare to examine whether attachment orientation may be most relevant to adaptive or maladaptive emotion-specific ER when in response to some forms of stress rather than others. There has been one previous study showing that attachment anxiety and avoidance are linked to ER differently depending on adolescents' reported emotions of sadness or anger (Brenning \& Braet, 2013). There is also some evidence that ER depends on the particular stressor (Amir, Horesh, \& LinStein, 1999; Charles \& Carstensen, 2008; Holmberg, Lomore, Takacs, \& Price, 2011; Roque et al. 2013), and there are theoretical reasons to expect that attachment may be more strongly linked to ER when stressful events are interpersonal in nature (e.g., rejection, conflict) rather than when stress is not (Besser \& Priel, 2009; Dewitte, De Houwer, Goubert, \& Buysse, 2010; Leary, Koch, \& HechenBleikner, 2001). Given this, participants in the present study were randomly assigned to one of two conditions, namely one condition where they were primed with an interpersonally stressful event prior to reporting ER and a second condition with a standard prime (i.e., using an existing standard form of an ER measure). Our purpose in this study was to examine whether attachment would correlate with emotion-specific ER under each condition in line with existing theory. We predicted that particular correlations with ER would emerge for each attachment style but that associations between attachment and ER would be stronger when ER was reported after relationship insecurity priming (i.e., after an interpersonally stressful event) than when there was no specific prime.

\section{Attachment and ER}

Attachment theory and ER. Building on classic developmental attachment theory (Bowlby, 1969; 1973; 1980), Shaver and Mikulincer (2002) explicated the links between attachment and psychopathology by developing a social psychological model 
focused on late adolescents' and adults' attachment and associations of attachment with ER strategies. During the period of late adolescence and early adulthood, attachment is increasingly transferred from parents to peers, as these needs become more fulfilled by peers and romantic partners (Fraley \& Davis, 1997). Research suggests mutual trust and caring close relationships facilitate the transfer and development of peer relationship attachment in this age group (Fraley \& Davis, 1997). In social psychological (Shaver \& Mikulincer, 2002) and developmental (Zimmer-Gembeck et al., 2016) theory, ER strategies are expected to be closely linked to attachment in late adolescence and early adulthood, with each attachment orientation expected to be associated with particular ways of regulating distress.

In most contemporary views of adolescent and adult attachment, secure attachment is differentiated from insecure attachment, including two types of insecurity - anxious and avoidant. As proposed in classic developmental attachment theory (Bowlby, 1973; 1980), and found in research on parent-child attachment (Brumariu, 2015; Brumariu, Kerns \& Seibert, 2012; Zimmer-Gembeck et al., 2016) and adult attachment (Mikulincer \& Shaver, 2007) secure individuals have been found to be more emotionally self-aware, and to display flexible emotion expression and regulation in conjunction with their tendency to expect that caregivers and others will respond sensitively. In contrast to other attachment orientations, individuals high in insecureanxious attachment tend to have a greater fear of rejection and abandonment (Brenning \& Braet, 2013). They also worry more intensely about the availability of the attachment figure for support and soothing, and they question their own value to the caregiver and others (Campbell \& Marshall, 2011). In adult attachment theory, individuals high in insecure-avoidant attachment do not feel as comfortable depending on others as they 
have a strong preference for emotional distance and self-reliance (Mikulincer \& Shaver, 2007), and there is also some support in children and adolescence for this association (Zimmer-Gembeck et al., 2016). Avoidant young people have also been found to minimise their generalised distress to avoid activating their attachment system (Cassidy, 1994).

We also expected specific differences in ER between attachment groups. Adult attachment theory suggests that individuals referred to as secure in their attachment orientation are expected to co-regulate distress and to have the capacity to cope more adaptively with their distress (Mikulincer, Shaver, \& Pereg, 2003). In contrast, insecurely attached individuals are expected to be less flexible in regulating their negative emotions as they would have reduced opportunities to learn appropriate ER strategies in their early attachment relationships. Even more specifically, individuals with an anxious attachment orientation are described as hyperactivating, whereby they have been found to emotionally overreact and attempt to elicit increased attention from others when distressed (Wei et al., 2005). In other words, they display more negative emotions and, often excessively, seek closeness to others when experiencing stress. Individuals with an avoidant attachment orientation have been described as deactivating, whereby they suppress negative emotions and distance themselves from others when distressed (Mikulincer et al., 2003). Deactivation means that those with avoidant tendencies would be more likely to respond to problems by discounting the severity of their feelings, and less often seeking or giving social support (Wei et al., 2005).

Overall, an insecure attachment orientation, either anxious or avoidant, is a risk factor for the development of psychopathology, and ER may help to explain these 
linkages (Kobak, Cassidy, Lyons-Ruth, \& Ziv, 2006). Studies have found that insecure attachment in adulthood can interfere with ER and effective coping, increasing the intensity of distress (Mikulincer \& Shaver, 2007). For example, adults classified as having an insecure attachment report less adaptive coping and emotional regulatory skills than those with secure attachment (Mikulincer et al., 2003). Secure attachment has been described as a protective factor that reduces the likelihood of ER difficulties and averts individuals from pathways leading to psychopathology.

Specific emotions and ER. Although Mikulincer and Shaver (2007) have described how attachment orientations will be associated with ER in particular ways, they often refer to distress and emotions in general, rather than to specific emotions. However, theory suggests ER may vary within a single individual as a function of specific emotion type. More specifically, functionalist theories of emotion suggest that specific emotions, and potentially ER of these specific emotions, serve important social functions, helping to maintain or disrupt relationships (e.g., Campos, Mumme, Kermoian, \& Campos, 1994). This approach suggests that by socialization and using emotion as feedback in interactions individuals learn how social partners will respond (DeOliveria, Bailey, Moran, \& Pederson et al., 2004). This is somewhat congruent with the differential emotions theory perspective of Izard (1990; 1991), who presumes that particular emotions are reflex-like. According to this theory each distress emotion has a distinct adaptive social function and elicits particular reactions from a social partner (Izard, 1991). Further, attachment literature suggests the experience of a specific emotion may differ and have different functions depending on an individual's attachment orientation goals. For example, a distinction can be made between functional anger (the anger of hope) used to discourage others' negative behaviour and maintain 
closeness by drawing attention, and dysfunctional anger (the anger of despair) that weakens relationships and tells others to leave (Bowlby, 1973; Mikulincer, 1998). Therefore, the social function of anger might not always be to tell others to leave, in some individuals it might be an attempt to force change in another person to maintain closeness.

Although these notions are still emerging, current approaches suggest that individuals would learn that expressions of sadness communicate a request for help and support from others, expressions of worry would communicate a need for help and protection from others, and expressions of anger either maintain closeness or tell others to leave (Brenning \& Braet, 2013). Applying this to the current ER model of attachment in close relationships, it would be expected that anxious attachment would be associated with heightening and dysregulation of all negative emotions (sadness, worry, and anger), to draw attention and elicit support. Avoidant attachment would be associated with sadness and worry suppression, and anger dysregulation, to maintain emotional distance from others. Secure attachment would be associated with lower dysregulation of all three emotions (sadness, worry, and anger), as such individuals would be able to regulate all emotions adaptively because they are comfortable and trusting of others as sources of support regardless of the particular distress feeling and context.

Studies of attachment and ER. Although very little research has been conducted, research in adults supports the idea that different attachment dimensions are associated with different ER strategies, usually assessed allowing participants to think about any self-identified situation and emotion (Mikulincer $\&$ Shaver, 2007). To date, however, only one study of ER strategies has focused on specific emotions (e.g., Brenning \& Braet, 2013). In this study attachment anxiety was related to dysregulation 
of sadness and anger. In contrast, attachment avoidance was related to suppression of sadness and dysregulation of anger. A limitation of this study was the focus on only attachment anxiety and avoidance neglecting to directly examine secure attachment. Thus, given that no previous study has examined specific ER of sadness, worry and anger and their associations with anxious, avoidant and secure attachment orientations in young adults, this was the first aim of this study.

\section{Attachment, ER, Stress, and Interpersonal Relationships}

Another extension on previous research was the use of relationship insecurity priming before participants completed the measure of ER. Attachment and the ability to explore in a relationship set the foundation for how to rely on people, and provide a strategy to cope when dealing with stress. During adolescence and emerging adulthood changes in stress perceptions occur, with increasing autonomy from parents, to new interests in friendships and romantic relationships (Sieffge-Krenke, 2011). In addition, adolescents and adults report that negative interpersonal events are the most common and most upsetting of stressful events (Spirito, Stark, Grace, \& Stamoulis, 1991). In one study, emerging adults reported between $46 \%$ and $82 \%$ of stressful events to be in interpersonal relationships involving parents, peers, and romantic partners (SeiffgeKrenke, Aunola, \& Nurmi, 2009). Research has also found that when young adults were asked to imagine that they were overhearing unpleasant social information about themselves they expressed more sadness and anger in response to the emotion provoking stimuli (Charles \& Carstensen, 2008). Furthermore, Leary et al. (2001) summarized decades of research illustrating the high distress associated with interpersonal rejection, and how emotions of sadness and worry are experienced when individuals feel like others are rejecting or do not accept them. Other research has found 
that anger is also an emotion that emerges among some individuals when they experience interpersonal stress, such as rejection (Zimmer-Gembeck \& Nesdale, 2013).

\section{The Present Study}

In summary, the primary aim of the present study was to test the ER model of attachment in close relationships (Mikulincer \& Shaver, 2007) when applied to specific emotions and within different stressful contexts. More specifically, the focus was on examining the associations between late adolescent and young adult attachment representations, and emotion specific (sadness, worry, and anger) ER. A further focus was on determining whether the associations between attachment and ER are stronger when participants were primed with relationship insecurity (read about an interpersonally stressful event) as compared to no priming (a general, unspecific stressor). Specific associations between attachment orientations and ER were expected, and differences in the strength of the associations were expected when comparing priming conditions:

H1 - There will be associations of anxious attachment with greater dysregulation of sadness, worry and anger, and attachment avoidance will be associated with greater dysregulation of anger (H1a). These associations will be stronger in the insecurity priming condition than without priming (H1b).

H2 - Attachment avoidance will be associated with more attempts to suppress sadness and worry (H2a). These associations will be stronger in the insecurity priming condition than without priming (H2b).

H3 - Attachment security will be associated with less dysregulation and suppression of all emotions (H3a). These associations will be stronger in the insecurity priming condition than without priming (H3b). 


\section{Method}

\section{Participants}

The participants were 383 young university students (181 men, 202 women) with a $M$ age of 19 years ( $S D=1.6$; between the ages of 16 to 23 ). Concerning family status, $68 \%$ of the participants reported that their parents were still married, whereas the remaining participants reported divorced or separated parents or the death of one parent. Concerning sociocultural background, $70 \%$ of participants were White/Caucasian, 19\% were Asian, 2\% were Aboriginal/Pacific Islander, the remaining 9\% identified as other.

\section{Procedure}

After receiving approval for this study from the university Human Subjects Review Committee, the participants in this study were recruited at random on a university campus in common use areas (87.5\%), and from a pool of first year psychology students (12.5\%). Participants completed a questionnaire administered in person on campus in classrooms or in common use areas. First year psychology students received course credit for participation. All other participants received a small chocolate bar on completion of the questionnaire.

There were two versions of the questionnaire allocated randomly to participants. The first version (completed by 193 participants, 91 young men and 102 young women) included a standard ER questionnaire without a specific stressor primed before completion. The second version (completed by 190 participants, 90 young men and 100 young women) primed the participants with the description of an interpersonal stressor prior to completing the ER measure. Questions about attachment were the same on the two versions of the survey and were completed before reading this vignette and answering the ER questions. 
The vignette used to prime interpersonal stress was adapted from a previous study (Charles \& Carstensen, 2008), which was shown to elicit anger and sadness among adults. The vignette used in the current study was as follows, "Imagine you are at a party with your best friend. You return from the bathroom and you overhear your best friend talking to another friend about you. As you approach they are discussing how boring you are and how badly you dress. Again, you know they are talking about you. You hear them comment about how you can't be trusted and they don't really like you. When they see you are standing right there, they stop talking immediately looking startled, and start making awkward conversation. Take a moment to think about how you would feel."

\section{Measures}

Attachment orientation. Participants completed the Experiences in Close Relationships Scale-Revised-General Short Form (ECR-R-GSF; Wilkinson, 2011), for the assessment of general relationship attachment anxiety and avoidance in adolescents and young adults. The anxiety scale (10 items) targets feelings of fear and abandonment and strong desires for closeness to others (e.g., "I find that other people don't want to be as close as I would like"). The avoidance scale (10 items) targets discomfort with closeness and dependence on others (e.g., I prefer not to show others how I feel deep down”). Items were rated on a 5-point scale from 1 (Strongly disagree) to 5 (Strongly agree). Cronbach's $\alpha$ s for the ECR-R-GSF in the current study were .86 and .79 for anxious and avoidant attachment, respectively.

Participants also completed an adolescent version of Bartholomew and Horowitz's (1991) Relationship Questionnaire (RQ). The RQ is a four-category 
attachment classification, which measures secure attachment, and insecure attachment (anxious, avoidant, and disorganised). Participants were presented with four descriptions of how people behave when with others and were asked to select the one that best describes them (e.g., I don't care if I am close to people. It is very important for me not to ask for help, because I like to do things on my own. I don't like it if people ask me for help). Participants were then asked to indicate on a scale from 0 (Not like me at all) to 6 (very much like me) how well each statement described them. For the purpose of this study ratings of secure, anxious, and avoidant were used.

ER. ER strategies were assessed using the ER inventory developed by Roth, Assor, Niemiec, Ryan, and Deci (2009), containing scales measuring dysregulation, suppression, and integration of ER. For this study, only the dysregulation scale (six items, e.g., "I often behave in a stressful or anxious way, even if I don't want to behave like that") and suppression scales (seven items, e.g., "I try to ignore feelings of stress or anxiety") were used. Items were adjusted to specifically refer to emotions of sadness, worry, and anger. For example, the item "I try to ignore feelings of stress or anxiety" was changed to "I try to ignore feelings of sadness/worry/anger." Responses to each item ranged from 1 (strongly disagree) to 5 (strongly agree). Cronbach's $\alpha$ s for the ER inventory in the current study were .91 and .87 for sadness suppression and dysregulation, .90 and .88 for worry suppression and dysregulation, and .89 and .88 for anger suppression and dysregulation, respectively.

\section{Overview of Analyses}

$M \mathrm{~s}, S D \mathrm{~s}$, and zero-order correlations between all measures were examined in preliminary analyses. In addition, $t$-tests were used to compare ER between the insecurity priming condition and the other condition. Next, to test all hypotheses, six 
standard multiple regressions, and follow-up moderation analyses were conducted. The six dependent variables (DVs) were dysregulation of sadness, dysregulation of worry, dysregulation of anger, suppression of sadness, suppression of worry, and suppression of anger. In these models, four independent variables (IVs) were entered to test Hypotheses 1a, 2a, and 3a. These included the three attachment orientations and condition, coded to compare the insecurity priming condition (1) to the standard condition (0). Path analysis using AMOS software was also conducted to examine all DVs and IVs in a single model. The results were almost identical to those found using the six regression models. Thus, we report only the regression models given that they provide a good representation of the findings. Interaction effects (e.g., secure attachment $\times$ condition) were then entered into each regression equation to test Hypotheses 1b, 2b, and 3b. Each attachment score was centred prior to forming the interaction term.

\section{Results}

\section{Missing Values and Nonnormality of Data}

There were 24 participants with missing data, with 16 missing only one item, and no participant missing more than six items. Because missing data were minimal and were at random, scores were formed based on the completed items to maintain all participants in the data analyses. An exception was five participants missing items on the RQ attachment measure; these missing values were estimated using single imputation.

The RQ attachment measure ratings for anxious and avoidant attachment displayed some positive skew. Bivariate correlations were examined before and after a square root transformation of these variables. There was minimal difference in the 
correlations. Thus, the untransformed attachment measures were maintained for all analyses.

\section{Randomisation Check}

To examine whether participants were successfully randomised to priming condition, their demographic characteristics were compared. There were no differences between the two conditions. Regarding age, there was no difference between the standard $(M=19.66, S D=1.60)$ and interpersonal stressor $(M=19.55, S D=1.57)$ conditions, $t(381)=.68, p=.50$. Also, the percentage of men and women completing each version did not differ, $\chi^{2}(1, N=383)=.002, p=.97$. There were also no differences in the proportion of white Australian versus other race/ethnicity in each condition, $\chi^{2}(1, N=383)=2.4, p=.12$, and no difference in the proportion with married versus other parent status, $\chi^{2}(1, N=383)=.05, p=.83$.

\section{Preliminary Analyses of Attachment Measures}

There were five continuous measures of attachment (ECR-R-GSF anxiety scale, ECR-R-GSF avoidance scale, and the three RQ description ratings) and one categorical measure of attachment (RQ category classification). The aims of the study focused on having a measure of each form of attachment. Thus, the multi-item subscales ECR-RGSF anxiety and ECR-R-GSF avoidance were used to validate the single item measure of each of the attachment orientations gathered via the RQ description ratings. The pattern of ratings and categories was consistent and validated the use of the single item measures as representative of attachment style. RQ anxious attachment was positively correlated with ECR-R-GSF anxiety $(r=.49)$. RQ secure attachment was negatively correlated with ECR-R-GSF anxiety and avoidance, $r=-.42$ and -.40, respectively. RQ avoidant attachment was positively correlated with ECR-R-GSF avoidance and to a 
lesser extent positively correlated with ECR-R-GSF anxiety, $r=.20$ and .12 , respectively. Therefore the RQ three description ratings were used for all further analysis.

\section{Zero-order Correlations between Measures}

Attachment and ER. $M$ s, $S D$ s, and bivariate correlations between the study variables are shown in Table 1. Providing preliminary support for Hypothesis 1a, individuals reporting higher levels of anxious attachment orientation were higher in emotion dysregulation for all emotions (sadness, worry, and anger). Also as predicted (Hypothesis 1a), individuals higher in avoidant attachment orientation were more likely to dysregulate anger, but avoidance was not associated with dysregulation of sadness or worry.

As predicted (Hypothesis 2a), an avoidant attachment orientation was positively correlated with suppression of sadness and worry (see Table 1). Also, individuals with higher anxious attachment orientations were more likely to suppress anger. Further, as predicted (Hypothesis 3a), a secure attachment orientation was negatively correlated with dysregulation of sadness, worry and anger, and negatively correlated with suppression of sadness and worry.

\section{Comparison of ER between the Interpersonal Stress Priming and Standard}

\section{Conditions}

Independent groups $t$-tests were used to compare ER responses between the insecurity priming and standard conditions. Regarding dysregulation, those in the interpersonal stressor condition $(M=2.80, S D=.88)$ reported more dysregulation of sadness than those in the standard condition $(M=2.50, S D=.91), t(381)=3.28, p=$ $.001, d=.34$. Also, those in the interpersonal stressor condition $(M=2.53, S D=.94)$ 
reported more dysregulation of anger than individuals assigned to the standard condition $(M=2.32, S D=.88), t(381)=2.29, p=.023, d=.23$. Finally, there was no difference in dysregulation of worry between conditions (Interpersonal $M=2.81, S D=.90$; Standard $M=2.70, S D=.95), t(381)=1.21, p=.23$. Regarding suppression of emotion, there was no difference in suppression of any emotion between conditions.

\section{Multivariate Analysis of Emotion Dysregulation, Attachment, and Priming}

\section{Condition}

Sadness. In the model of sadness dysregulation, anxious attachment, $\beta=.32, \mathrm{p}$ $<.001$, and condition, $\beta=.19, \mathrm{p}<.001$, were significantly associated with more sadness dysregulation, and secure attachment, $\beta=-.11, \mathrm{p}<.05$, was significantly associated with less sadness dysregulation (see Table 2). Overall, $20.4 \%$ of the variance was accounted for by the IVs, $F(4,378)=24.28, p<.001$. When moderator terms were examined in Step 2 of this model, no interaction between attachment and condition was significantly associated with sadness dysregulation (anxious attachment $\times$ condition $F(1,377)=.02, p=.88 ;$ secure attachment $\times$ condition $F(1,377)=.77, p=.38 ;$ avoidant attachment $\times$ condition $F(1,377)=3.33, p=.07)$. These findings indicated that the association between attachment orientation and sadness dysregulation did not differ depending on the priming condition.

Worry. In the model of worry dysregulation, anxious attachment, $\beta=.36, \mathrm{p}<$ .001 , was significantly associated with more worry dysregulation, and secure attachment, $\beta=-.13, \mathrm{p}<.05$, was significantly associated with less worry dysregulation (see Table 2). Overall, $17.8 \%$ of the variance was accounted for by the IVs, $F(4,378)=$ $20.43, p<.001$. When moderator terms were examined in step 2 of this model, no interaction between attachment and condition was significantly associated with worry 
dysregulation (anxious attachment $\times$ condition $F(1,377)=.01, p=.93$; secure attachment $\times$ condition $F(1,377)=.70, p=.40 ;$ avoidant attachment $\times$ condition $F(1$, $377)=3.25, p=.07)$.

Anger. In the model of anger dysregulation, avoidant attachment, $\beta=.19, \mathrm{p}<$ .001 , anxious attachment, $\beta=.18, \mathrm{p}<.001$, and condition, $\beta=.13, \mathrm{p}<.01$, were significantly associated with more anger dysregulation, and secure attachment, $\beta=-.18$, $\mathrm{p}<.001$, was significantly associated with less anger dysregulation (see Table 2 ). Overall, $12.7 \%$ of the variance was accounted for by the IVs, $F(4,378)=13.76, p<$ .001 . When moderator terms were examined in step 2 , no interaction between attachment and condition was significantly associated with anger dysregulation (anxious attachment $\times$ condition $F(1,377)=1.57, p=.21$; secure attachment $\times$ condition $F(1$, $377)=2.77, p=.10$; avoidant attachment $\times$ condition $F(1,377)=2.74, p=.10)$.

\section{Emotion Suppression, Attachment, and Stressor Condition}

Sadness. In the model of sadness suppression, avoidant attachment was significantly associated with more suppression of sadness, $\beta=.18, \mathrm{p}<.001$, and secure attachment was significantly associated with less suppression of sadness, $\beta=-.16, \mathrm{p}<$ .01 (see Table 3$)$. Overall, $6.5 \%$ of the variance was accounted for by the IVs, $F(4,378)$ $=6.59, p<.001$. When moderator terms were examined, one significant moderator effect was found; avoidant attachment $\times$ condition accounted for a significant $1.3 \%$ of the variance to the explanation of sadness suppression, $F(1,377)=5.47, p=.02$. This finding indicates that the association between avoidant attachment and sadness suppression, differed significantly depending on stressor condition (standard or interpersonal). The other two moderator terms were not significant, anxious attachment 
$\times$ condition $F(1,377)=1.84, p=.18$; secure attachment $\times$ condition $F(1,377)=1.86, p$ $=.17$.

Simple slopes analysis (see Figure 1) indicated there was a significant positive association between avoidant attachment and sadness suppression among participants in the insecurity priming condition, $t(377)=4.13, p<.001$. For participants in the standard condition there was no significant association between avoidant attachment and sadness suppression $t(377)=0.98, p=.33$.

Worry. In the model of worry suppression, avoidant attachment, was significantly associated with more suppression of worry, $\beta=.11, \mathrm{p}<.05$, and secure attachment was significantly associated with less suppression of worry, $\beta=-.14, \mathrm{p}<.01$ (see Table 3). Overall, $4.2 \%$ of the variance was accounted for by the IVs, $F(4,378)=$ $4.15, p=<.01$. When moderator terms were examined in step 2 of this model, no interaction between attachment and condition was significantly associated with worry suppression (anxious attachment $\times$ condition $F(1,377)=3.25, p=.07$; secure attachment $\times$ condition $F(1,377)=2.56, p=.11$; avoidant attachment $\times$ condition $F(1$, $377)=.55, p=.46)$.

Anger. In the model of anger suppression, anxious attachment, $\beta=.15, \mathrm{p}<.01$, was significantly associated with more anger suppression (see Table 3). Overall, $2.6 \%$ of the variance was accounted for by the IVs, $F(4,378)=2.50, p=.04$. When moderator terms were examined, no interaction between attachment and condition was significantly associated with anger suppression, anxious attachment $\times$ condition $F(1$, $377)=3.01, p=.08$; secure attachment $\times$ condition $F(1,377)=.84, p=.36$; avoidant attachment $\times$ condition $F(1,377)=1.36, p=.25$. 


\section{Discussion}

There are many classic developmental (Bowlby, 1969), contemporary developmental (Ainsworth, 1989; Cassidy, 1994; Cassidy et al., 2009; Main, 2000; Sroufe, 1996), and adolescent- or adult-focused social psychological (Mikulincer \& Shaver, 2007; Shaver \& Mikulincer, 2002) theories that make specific hypotheses regarding how secure attachment should benefit ER, and anxious and avoidant attachment orientations should be associated with maladaptive patterns of ER, including emotion dysregulation and suppression. Moreover, theory and previous research suggest that attachment should play a role in ER across many contexts, but that the specific emotions under regulation and the context should be important to consider (Brenning \& Braet, 2013; Roque et al., 2013). In the present study we predicted that attachment orientation would have different associations with sadness and worry compared to anger dysregulation and suppression, and a context that primes insecurity (in this case an interpersonal stressor that threatens rejection and dislike) would be more challenging for regulation (as compared to a "standard" condition) among those without a secure attachment orientation. However, no previous study had examined these notions directly. Thus, the study reported here is the first investigation of three attachment orientations as correlated with emotion-specific ER under the condition of relationship insecurity priming compared to a standard ER measurement condition.

\section{Insecure Attachment and ER}

Anxious attachment. In support of theory (Shaver and Mikulincer, 2002), and consistent with predictions, the results from our multivariate analyses considering all attachment orientations simultaneously showed that individuals with higher anxious attachment orientation scores reported greater dysregulation of sadness, worry, and 
anger. Thus, despite only using a single item measure of attachment anxiety, individuals who report a more elevated anxious attachment orientation report heightened dysregulation of sadness, worry and anger when under stress. As proposed in theory (Shaver \& Mikulincer, 2002) and found in previous research (Brenning \& Braet, 2013) their emotions may appear hyperactivated partly because they have difficulty regulating them. However, this dysregulation of emotion may be one available resource for gaining support from others, given that emotional displays of sadness and worry can send a message of the need for social support and assistance. Emotion theories suggest that overt displays of sadness, worry, and anger can be beneficial to elicit increased attention from others to maintain closeness when distressed (Wei et al., 2005).

In addition to more anger dysregulation, anxious attachment was associated with more anger suppression, which was not expected. This finding is consistent with the anxious attachment profile of responses to stress, with anxiously attached individuals dysregulation of anger coupled with a heightened use of suppression. Future research could benefit from investigating the associations between distinct types or functions of anger (Bowlby, 1973; Mikulincer, 1998), attachment orientations, and emotion regulation.

Avoidant attachment. The results from our multivariate analyses considering all attachment orientations simultaneously showed that individuals higher in attachment avoidance reported more anger dysregulation, as predicted. Also, associations of avoidant attachment orientation with sadness and worry dysregulation were not significant. These findings conform to functionalist and differential emotion theories (e.g., Campos et al., 1994; Izard, 1991) and are consistent with previous research that found different links between avoidant attachment and ER strategies depending on 
emotion type (e.g., Brenning \& Braet, 2013). For example, as avoidant individuals prefer emotional distance they are expected to dysregulate anger but not sadness or worry. Such a specific dysregulation pattern may serve the function of avoiding closeness with others (Brenning \& Braet, 2013; Campos et al., 1994).

Also consistent with the expected pattern of ER among individuals higher in avoidant attachment orientation, individuals who reported that they were more avoidant reported more sadness suppression and more worry suppression. One possible explanation aligns to functionalist and differential emotion theories (e.g., Campos et al., 1994; Izard, 1991), which outline how individuals learn expressions of sadness to communicate a request for support, and expressions of worry to communicate a need for protection from others. In this way, avoidant attached individuals may have greater access to deactivating strategies to avoid drawing attention and support from others and may suppress sadness and worry to maintain emotional distance (e.g., Brenning \& Braet, 2013).

Anger dysregulation in anxious and avoidant attachment. The consistent finding for higher levels of anger dysregulation when participants reported higher levels of anxious and avoidant attachment orientation is interesting considering the different motivations of each orientation, to seek closeness or maintain distance, respectively. These results imply support for distinct types of anger or different functions of anger, as described by Bowlby (1973) and Mikulincer (1998). The motivations of anxiously attached individuals align with dysregulation of functional anger, which discourages negative behaviour and maintains closeness by drawing others' attention. In contrast, the motivations of individuals who have a heightened avoidant attachment orientation align with dysregulation of dysfunctional anger, which distances others and weakens 
relationships (Bowlby, 1973; Mikulincer, 1998). These proposed associations provide one possible explanation for the associations found between anxious and avoidant attachment and anger dysregulation.

\section{Secure Attachment and ER}

Substantial support was found for the hypothesis that individuals reporting greater security of attachment would have a less negative profile of maladaptive ER across all emotions. The results from our multivariate analyses considering all attachment orientations simultaneously showed that participants higher in security reported significantly less dysregulation of sadness, worry and anger, and less sadness and worry suppression. Thus, there were many unique benefits of security for ER dysregulation and suppression once insecure attachment orientations were considered.

This finding is consistent with attachment theory (Bowlby, 1973; 1980) where secure individuals are expected to display more adaptive ER in conjunction with expecting others will respond sensitively; findings also concur with parent-child attachment and ER research (Brumariu, 2015; Brumariu, Kerns \& Seibert, 2012; Zimmer-Gembeck et al., 2016) and adult attachment theory and research (Mikulincer \& Shaver, 2007). In addition the general protective nature of security is consistent with previous research (Zimmer-Gembeck et al., 2016) and this new finding regarding specific emotions suggests attachment security is protective against ER difficulties for specific emotions. The finding that secure attachment was not associated with less anger suppression was unexpected, however. One possible explanation is that securely attached individuals may experience anger differently compared to insecure individuals. Secure attachment may be associated with a tendency to experience anger as an incentive system rather than aversive system (Carver \& Harmon-Jones, 2009). In this 
view anger translates to motivation, where anger motivates an individual to overcome an obstacle on the way to reaching a goal (Carver \& Harmon-Jones, 2009). Therefore secure individuals have no need to supress anger as rather than being an inappropriate emotion they may have greater capacity to channel their anger in a more adaptive and positive way.

\section{Attachment, ER, Stress, and Interpersonal Relationships}

The third aim of this study addressed the particular salience of attachment theory and the ER model for understanding emotion-specific ER in context. It was predicted that ER would be more strongly associated with attachment orientation after priming participants with insecurity before answering questions about ER, rather than using the standard ER measurement approach (i.e., with no prime for the type of context or stressor). This prediction was based on theory suggesting a stronger activation of the attachment system in times of interpersonal relationship stress that results in insecurity (Bowlby, 1969; 1973; 1980). Although participants reported more sadness and anger dysregulation following the insecurity prime as compared to the standard no prime condition, there were mixed findings regarding moderation by condition (i.e., context). There was no support for moderation by condition when investigating ER dysregulation, and very partial support when investigating ER suppression.

More specifically, the results from our moderation analysis showed that the association between avoidant attachment and sadness suppression was stronger when ER was reported following an insecurity prime than when ER was reported without a prime. As an explanation for their higher level of sadness suppression in the insecurity prime condition, the sadness associated with interpersonal situations may be particularly 
distressing for avoidant individuals due to their discomfort with close interpersonal situations that threaten their need to maintain independence.

Taken together, the effect of insecurity priming was quite limited. One explanation for this finding, as well as the finding that most associations between attachment and ER did not differ by condition, is the limitation of using a no prime as a comparison to an insecurity prime. By allowing participants to complete the ER measure as is usually done (without a prime for context), participants might have been predominantly focusing on situations that are interpersonal in nature, given that interpersonal difficulties are one of the most common and salient stressors reported by young adults (Charles \& Carstensen, 2008; Seiffge-Krenke et al., 2009; Spirito et al., 1991). In future research, it would be useful to specify a context for all participants to allow for more direct comparisons of ER between contexts, and differences in associations of attachment with ER in different contexts.

\section{Limitations, Future Research, and Conclusion}

Several other limitations of the present study should be acknowledged. First, because the participants were university students, the generalizability of findings may be limited. However, university students in Australia come from a wide range of backgrounds, as shown in the demographic characteristics reported here. Second, all measures were self-reported. Although the reliability and validity of all of the items used here were established in past research, social desirability must be considered, and there is the possibility that associations were inflated due to shared method variance. Finally, only one item was used to measure each attachment orientation. Although these items were based on a widely used measure, and were validated against a more 
extensive measure of attachment avoidance and anxiety, single item measures can be limited and no reliability information is available for them.

In conclusion, a key finding of the present study was that individuals' attachment representations were associated with ER dysregulation and suppression for most emotions, with different patterns found for secure as compared to the two insecure attachment orientations as predicted. It is important to consider sadness, worry, and anger ER separately when examining how these may emerge from social relationship histories and current models of relationships with others. These findings stress the importance of focusing on all attachment styles in association with emotion-specific $\mathrm{ER}$, to gain a comprehensive understanding of emotional functioning. Yet, the context within which ER is required may not be an important consideration when examining how attachment and ER are associated, given that we found little evidence of stronger associations between attachment and ER following an insecurity prime than without any prime. 


\section{References}

Ainsworth, M. D. S. (1989). Attachments beyond infancy. American Psychologist, 44, 709-716. doi:10.1037/0003-066X.44.4.709

Amir, M., Horesh, N., \& Lin-Stein, T. (1999). Infertility and adjustment in women: The effects of attachment style and social support. Journal of Clinical Psychology in Medical Settings, 6, 463-479. doi:10.1023/A:1026280017092

Bartholomew, K., \& Horowitz, C. M. (1991). Attachment styles among young adults: A test of a four category model. Journal of Personality and Social Psychology, 61, 226-244. doi:10.1037/0022-3514.61.2.226

Besser, A., \& Priel, B. (2009). Emotional responses to a romantic partner's imaginary rejection: The roles of attachment anxiety, covert narcissism, and selfevaluation. Journal of Personality, 77, 287-325. doi:10.111/j.14676494.2008.00546.x

Bowlby, J. (1969). Attachment and loss: Vol. 1. London, UK: Hogarth P.

Bowlby, J. (1973). Attachment and loss: Vol. 2. Separation: Anxiety and anger. New York, NY: Basic Books.

Bowlby, J. (1980). Attachment and loss: Vol. 3. Sadness and depression. New York, NY: Basic Books.

Brenning, K. M., \& Braet, C. (2013). The emotion regulation model of attachment: An emotion specific approach. Personal Relationships, 20, 107-123. doi:10.111/j.1475-6811.2012.01399.x

Brumariu, L. E. (2015). Parent-child attachment and emotion regulation. In G. Bosmans \& K. A. Kerns (Eds.), Attachment in middle childhood: Theoretical 
advances and new directions in an emerging field. New Directions for Child and Adolescent Development, 148, 31-45.

Brumariu, L. E., Kerns, K. A., \& Seibert, A. (2012). Mother-child attachment, emotion regulation, and anxiety symptoms in middle childhood. Personal Relationships, 19, 569-585. doi:10.1111/j.1475-6811.2011.01379.x

Campbell, L., Marshal, T. (2011). Anxious attachment and relationship processes: An interactionist perspective. Journal of Personality, 79, 1219-1249. doi:10.1111/j.1467-6494.2011.00723.x

Campos, J. J., Mumme, D. L., Kermoian, R., \& Campos, R. G. (1994). A functionalist perspective on the nature of emotion. Monographs of the Society for Research in Child Development, 59, 284-303. doi:10.2307/1166150

Carver, C. S., \& Harmon-Jones, E. (2009). Anger is an approach-related affect: Evidence and implications. Psychological Bulletin, 135, 183-204. doi:10.1037/a0013965

Cassidy, J. (1994). Emotion regulation: Influences of attachment relationships. Monographs of the Society for Research in Child Development, 59, 228-249. doi: $10.2307 / 1166148$

Cassidy, J., Shaver, P. R., Mikulincer, M., \& Lavy, S. (2009). Experimentally induced security influences responses to psychological pain. Journal od Social and Clinical Psychology, 28, 463-478. doi:10.1521/jscp.2009.28.4.463

Charles, S. T., \& Carstensen, L. L. (2008). Unpleasant situations elicit different emotional responses in younger and older adults. Psychol Aging, 23, 495-504. doi:10.1037/a0013284 
Cooper, M. L., Shaver, P. R., \& Collins, N. L. (1998). Attachment styles, emotion regulation, and adjustment in adolescence. Journal of Personality and Social Psychology, 74, 1380-1397. doi:10.1037/0022-3514.74.5.1380

DeOliveira, C. A., Bailey, H. N., Moran, G., \& Pederson, D. R. (2004). Emotion socialization as a framework for understanding the development of disorganized attachment. Social Development, 13, 437-467. doi:10.111/j.14679507.2004.00276.x

Dewitte, M., De Houwer, J., Goubert, L., \& Buysse, A. (2010). A multi-modal approach to the study of attachment-related distress. Biological Psychology, 85, 149-162. doi:10.1016/j.biopsycho.2010.06.006

Fraley, R. C., \& Davis, K. E. (1997). Attachment formation and transfer in young adults' close friendships and romantic relationships. Personal Relationships, 4, 131-144. doi:10.1111/j.1475-6811.1997.tb00135.x

Holmberg, D., Lomore, C. D., Takacs, T. A., \& Price, E. L. (2011). Adult attachment styles and stressor severity as moderators of the coping sequence. Personal Relationships, 18, 502-517. doi:10.1111/j.1475-6811.2010.01318.x

Izard, C. E. (1990). Facial expressions and the regulation of emotions. Journal of Personality and Social Psychology, 58, 487-498. doi:10.1037/00223514.58.3.487

Izard, C. E. (1991). The psychology of emotions. New York, NY: Plenum.

Kobak, R., Cassidy, J., Lyons-Ruth, K., \& Ziv, Y. (2006). Attachment, stress, and psychopathology: A developmental pathways model. In D. Cicchetti \& D. J. Cohen (Eds.), Developmental psychopathology (pp. 333-369). New Jersey: John Wiley \& Sons. 
Kopp, C. B. (1989). Regulation of negative emotions: A developmental view. Developmental Psychology, 25, 343-354. doi: 10.1037/0012-1649.25.3.343

Leary, M. R., Koch, E. J., \& HechenBleikner, N. R. (2001). Emotional responses to interpersonal rejection. In M. Leary (Eds.), Interpersonal rejection (pp. 145166). Cary, NC: Oxford University Press.

Main, M. (1996). Introduction to the special section on attachment and psychopathology: 2. Overview of the field of attachment. Journal of Consulting and Clinical Psychology, 64, 237-243. doi:10.1037/0022-006X.64.2.237

Main, M. (2000). Attachment theory. In A. E. Kazdin (Ed.), Encyclopedia of Psychology, Vol 1 (pp. 289-293). New York: Oxford University Press.

Mikulincer, M. (1998). Adult attachment style and individual differences in functional versus dysfunctional experiences of anger. Journal of Personality and Social Psychology, 74, 513-524. doi:10.1037/0022-3514.74.2.513

Mikulincer, M., \& Shaver, P. R. (2007). Attachment in adulthood: Structure, dynamics, and change. New York, NY: Guilford.

Mikulincer, M., Shaver, P. R., \& Pereg, D. (2003). Attachment theory and affect regulation: The dynamics, development, and cognitive consequences of attachment-related strategies. Motivation and Emotion, 27, 77-102. doi:10.1023/A:1024515519160

Roque, L., Veríssimo, M., Fernandes, M., \& Rebelo, A. (2013). Emotion regulation and attachment: Relationships with children's secure base, during different situational and social contexts in naturalistic settings. Infant Behaviour and Development, 36, 298-306. doi:10.1016/j.infbeh.2013.03.003 
Roth, G., Assor, A., Niemiec, C. P., Ryan, R. M., \& Deci, E. L. (2009). The emotional and academic consequences of parental conditional regard: Comparing conditional positive regard, conditional negative regard, and autonomy support as parenting practices. Developmental Psychology, 45, 1119-1142. doi:10.1037/a0015272

Seiffge-Krenke, I. (2011). Coping with relationship stressors: A decade review. Journal of Research on Adolesence, 21, 196-210. doi:10.1111/j.1532-7795.2010.00723.x

Seiffge-Krenke, I., Aunola, K., \& Nurmi, J. (2009). Changes in stress perception and coping during adolescence: The role of situational and personal factors. Child Devepopment, 80, 259-279. doi:10.1111/j.1467-8624.2008.01258.x

Shaver, P. R., \& Mikulincer, M. (2002). Attachment related psychodynamics. Attachment and Human Development, 4, 133-161. doi:10.1080/14616730210154171

Spirito, A., Stark, L. J., Grace, N., \& Stamoulis, D. (1991). Common problems and coping strategies reported in childhood and early adolescence. Journal of Youth and Adolescence, 20, 531-544. doi:10.1007/BF01540636

Sroufe. A. L. (1996). Emotional development: The organization of emotional life in the early years. New York: Cambridge University Press.

Thompson, R. A. (1994). Emotion regulation: A theme in search of definition. Monographs of the Society for Research in Child Development, 59,25-52. doi:10.1111/j.1540-5834.1994.tb01276.x

Wei, M., Vogel, D. L., Ku, T.-Y., \& Zakalik, R. A. (2005). Adult attachment, affect regulation, negative mood, and interpersonal problems: The mediating roles of 
emotional reactivity and emotional cutoff. Journal of Counseling Psychology, 52, 14-24. doi:10.1037/0022-0167.52.1.14

Wilkinson, R. B. (2011). Measuring attachment dimensions in adolescents:

Development and validation of the experiences in close relationships- RevisedGeneral short form. Journal of Relationships Research, 2, 53-62. doi:10.1375/jrr.2.1.53

Zimmer-Gembeck, M. J., \& Nesdale, D. (2013). Anxious and angry rejection sensitivity, social withdrawal, and retribution in high and low ambiguous situations. Journal of Personality, 81, 29-38. doi:10. 1111/j.14676494.2012.00792.x

Zimmer-Gembeck, M. J., Webb, H. J., Pepping, C. A., Swan, K., Merlo, O., Skinner, E. A., Avdagic, E., \& Dunbar, M. (2016). Review: Caregiver-child attachment, emotion regulation, and coping with stress. International Journal of Behavioral Development. 


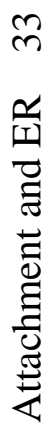

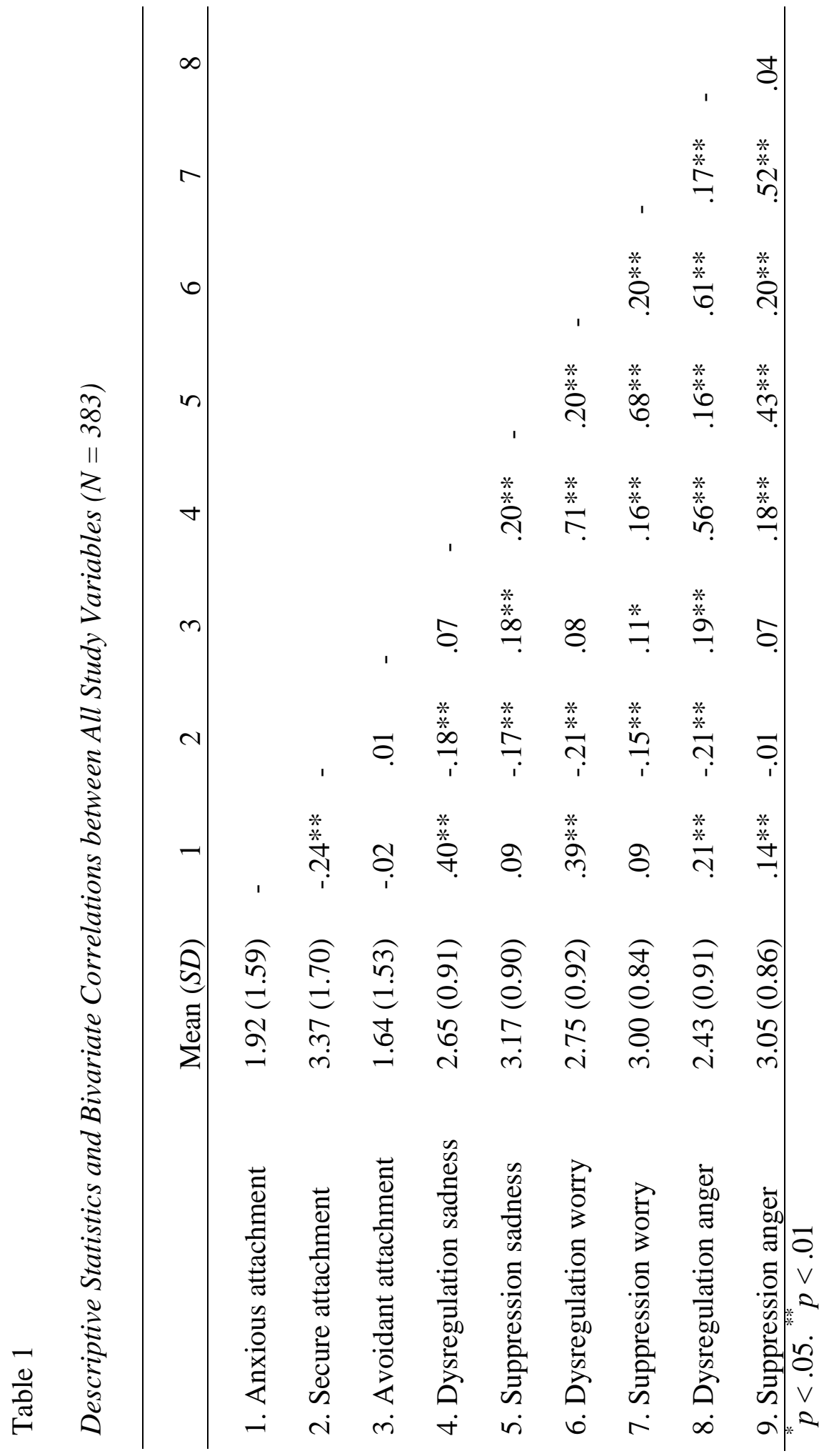




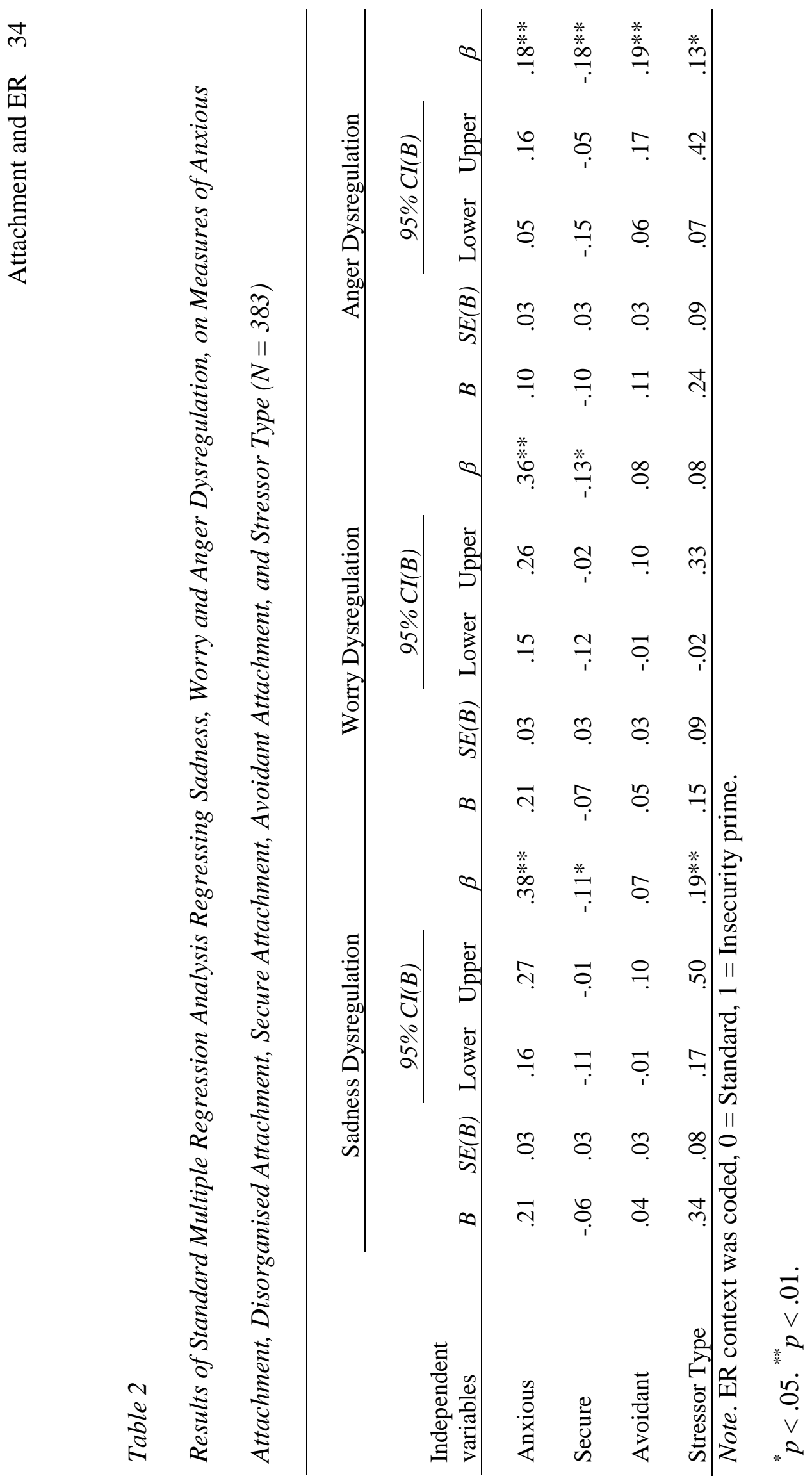




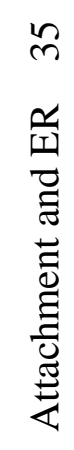

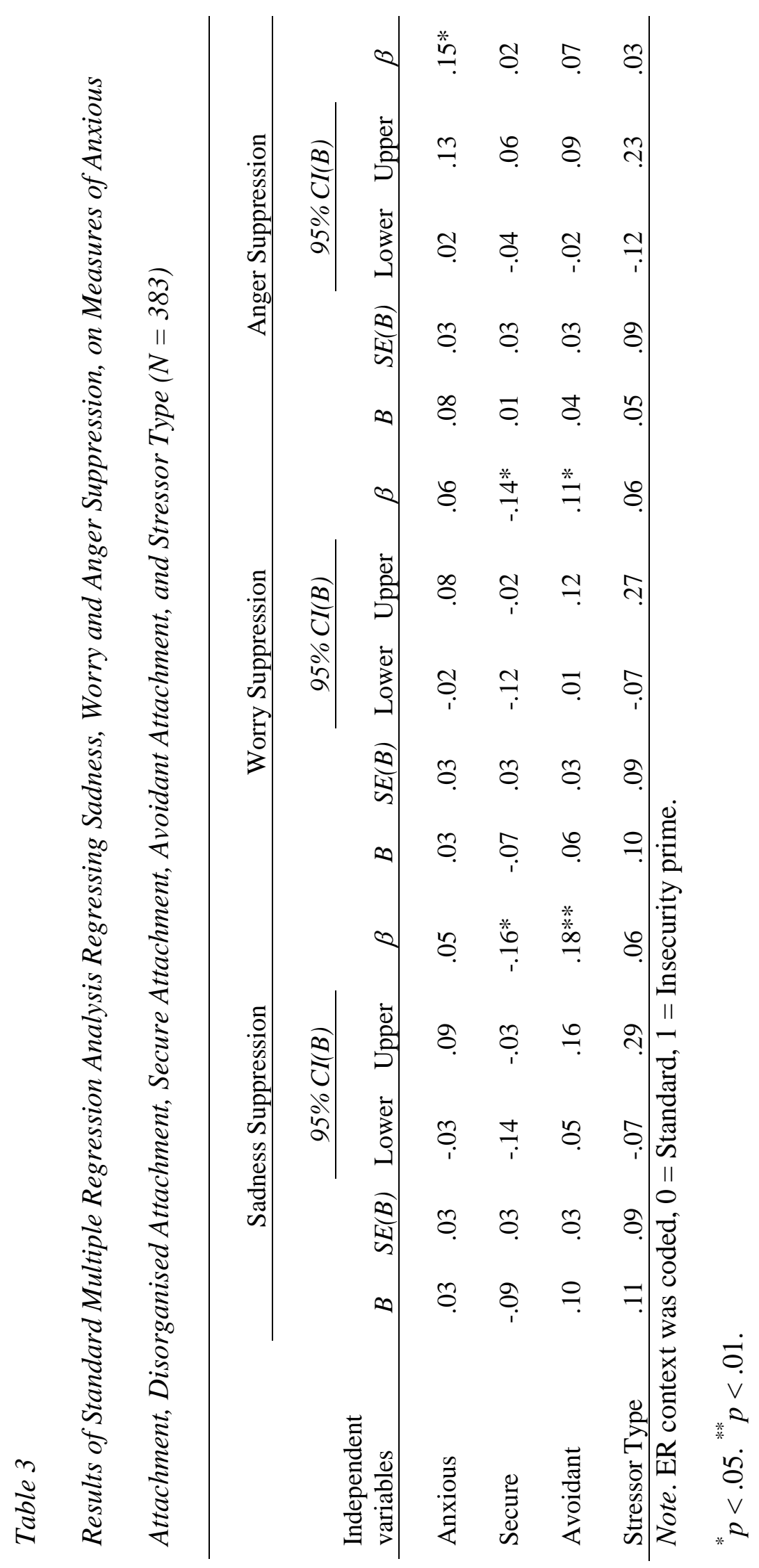




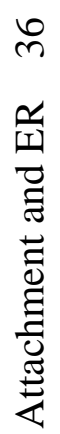

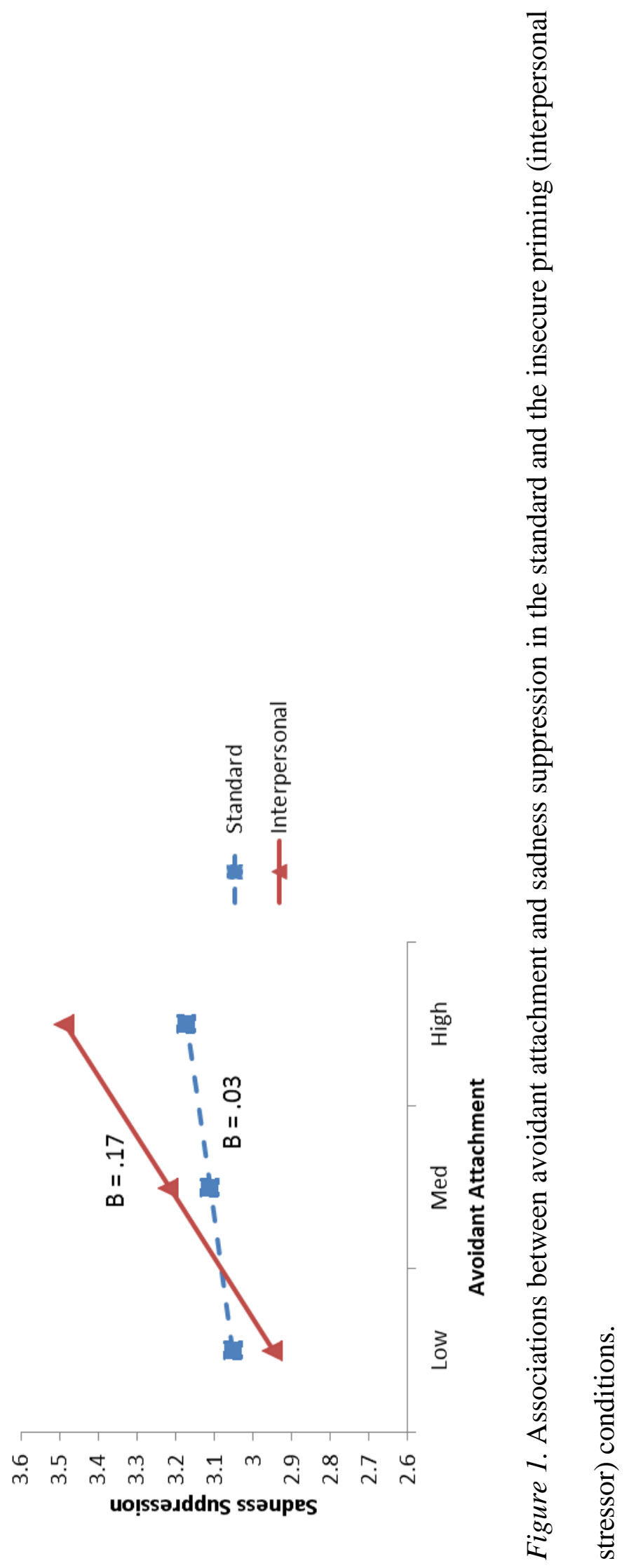

